\title{
Determinación de la digestibilidad y energía digestible del forraje seco de mucuna (Mucuna pruriens) en cuyes
}

\author{
Determination of digestibility and digestible energy of dry velvet \\ (Mucuna pruriens) forage in guinea pigs
}

\author{
Alejandrina Sotelo M. ${ }^{1,7}$, Rocio Valenzuela R. ${ }^{1}$, Mary Flor Césare C. ${ }^{2}$, \\ Cecilia Alegría A. ${ }^{2}$, Edgar Norabuena M. ${ }^{3}$, Teresa Gonzáles H. ${ }^{4}$, Elizabeth Paitan A. \\ Maria Teresa Valderrama R. ${ }^{6}$, Mariano Echevarría R. ${ }^{1}$
}

\section{Resumen}

\begin{abstract}
El estudio tuvo como objetivo determinar los coeficientes de digestibilidad y la energía digestible del forraje seco de mucuna (Mucuna pruriens). Se empleó el método indirecto, considerando como dieta basal el concentrado utilizado en la Granja de Cuyes de Cieneguilla (Lima, Perú). El experimento tuvo una fase de adaptación (14 días) y otra de colección de heces ( 5 días). Se utilizaron 10 cuyes machos adultos de tres meses de edad, distribuidos en forma aleatoria en jaulas metabólicas individuales. Cinco animales fueron alimentados con la dieta basal y los otros cinco con la dieta experimental: $80 \%$ dieta basal y $20 \%$ forraje seco de mucuna. Los coeficientes de digestibilidad aparente obtenidos para la mucuna fueron: $66.29 \%$ de materia seca, $66.13 \%$ de materia orgánica, $74.02 \%$ de proteína cruda, $50.82 \%$ de fibra cruda, $60.18 \%$ de extracto etéreo, $78.22 \%$ de extracto libre de nitrógeno y $73.33 \%$ de ceniza. El valor de energía digestible estimado fue de $2.61 \mathrm{kcal} / \mathrm{g}$.
\end{abstract}

Palabras clave: digestibilidad, energía digestible, cuyes, forraje seco, Mucuna pruriens

\section{Abstract}

This study aimed to determine the apparent digestibility coefficients and digestible energy of dry velvet bean fodder (Mucuna pruriens). The indirect method was used, considering as a basal diet the concentrate used in the university guinea pig farm (Lima,

\footnotetext{
${ }^{1}$ Facultad de Zootecnia, Universidad Nacional Agraria La Molina, Lima, Perú

${ }^{2}$ Facultad de Ciencias, Universidad Nacional Agraria La Molina, Lima, Perú

${ }^{3}$ Facultad de Ingeniería Química y Textil, Universidad Nacional de Ingeniería, Lima, Perú

${ }^{4}$ Facultad de Ciencias de Ingeniería, Universidad Nacional de Huancavelica, Huancavelica, Perú

${ }^{5}$ Facultad de Ingenierías Alimentarias, Universidad Nacional del Centro del Perú, Huancayo, Perú

${ }^{6}$ Facultad de Ingeniería Ambiental y Recursos Naturales, Universidad Nacional del Callao, Lima, Perú

${ }^{7}$ E-mail: asotelo@lamolina.edu.pe
}

Recibido: 26 de enero de 2019

Aceptado para publicación: 23 de diciembre de 2019

Publicado: 31 de marzo de 2020 
Peru). The experiment had an adaptation phase (14 days) and a faecal collection phase (5 days). Ten adult male 3 -month old guinea pigs randomly distributed in individual metabolic cages were used. Five animals were fed the basal diet and the other five with the experimental diet: $80 \%$ basal diet and $20 \%$ dry velvet bean fodder. The apparent digestibility coefficients obtained for the dry velvet bean were: $66.29 \%$ dry matter, $66.13 \%$ organic matter, $74.02 \%$ crude protein, $50.82 \%$ crude fibre, $60.18 \%$ ether extract, $78.22 \%$ nitrogenfree extract and $73.33 \%$ ash. The estimated digestible energy value was $2.61 \mathrm{kcal} / \mathrm{g}$.

Key words: digestibility, digestible energy, guinea pig, dry forage, Mucuna pruriens

\section{INTRODUCCIÓN}

Los sistemas de producción están condicionados por los costos de producción y, dentro de ellos, por el factor alimentación (Narváez y Delgado, 2012). En la crianza de cuyes, las especies forrajeras de mayor uso, tanto como forraje fresco o como ingrediente dentro del concentrado, son el maíz chala y la alfalfa.

El cultivo de maíz requiere una elevada inversión en fertilizantes, mientras que la alfalfa tiene altos costos de mantenimiento. En este contexto, surge la necesidad de encontrar nuevas alternativas que puedan sustituir a estos dos cultivos tradicionales y que posean similares características nutritivas, un menor costo de producción, así como también que permita el desarrollo sostenible e integrado de la actividad agrícola y pecuaria. El cultivo que cumple con estas características es la mucuna (Stizolobium deeringianum), leguminosa tropical que ha sido cultivada con éxito en zonas tropicales, demostrando alto rendimiento productivo y nutritivo, buena aceptabilidad por los animales, así como por su capacidad para incorporar materia orgánica al suelo e inhibir el crecimiento de las malas hierbas (Ruiz et al., 2015).

La mucuna se usa como abono verde, como cultivo de cobertura para controlar malezas, como forraje o como banco de pro- teína o concentrado (Peters et al., 2010). En la Selva Baja del Perú, especialmente en Loreto, el cultivo de la fabácea «Mucuna», se convierte en una alternativa de mejora de la producción pecuaria por su adaptación y su buena calidad (Caro, 2013). El forraje de mucuna tiene una composición química y física diferente al de otras especies, de allí que su utilización como fuente de energía puede provocar modificaciones en la ecología del intestino de los animales que lo consumen (Rodríguez et al., 2012). Desde el punto de vista químico se caracteriza por tener un mayor contenido de materia seca, proteína cruda, fibra cruda y grasa y menor contenido de pared celular y lignina. En lo referente a la composición física, tiene mayor solubilidad, capacidad buffer y menor capacidad de absorción de agua (Martínez et al., 2008).

A pesar de ser una excelente alternativa en la alimentación de cuyes, todavía no se han realizado estudios in vivo para obtener los coeficientes de digestibilidad, el cual es uno de los métodos más importantes de evaluación nutritiva (McDonald, 2013). La digestibilidad es una medida de la disponibilidad biológica de nutrientes y es importante en la formulación de una ración balanceada. Solo la parte que es soluble o que se convierte en soluble por hidrólisis u otros cambios químicos o físicos pueden ingresar a la circulación y ser usado por el animal como materiales de construcción o fuente de energía (Caprita et al., 2013). En el presente estudio se determinaron los coeficientes de digesti- 
bilidad y estimó la energía digestible y nutrientes digestibles totales del forraje seco de mucuna (Mucuna pruriens) a través de ensayos de digestibilidad in vivo en cuyes.

\section{Materiales y Métodos}

\section{Localización del Estudio}

El trabajo experimental se llevó a cabo en las instalaciones del Laboratorio de Evaluación Biológica de Alimentos y los análisis químicos se realizaron en el Laboratorio de Evaluación Nutricional de Alimentos (LENA), ambos correspondientes al Departamento Académico de Nutrición de la Facultad de Zootecnia, Universidad Nacional Agraria La Molina UNALM), en Lima, Perú.

\section{Animales}

Se usaron 10 cuyes machos de línea mejorada Tipo 1, de tres meses de edad, con peso promedio de $854.2 \mathrm{~g}$, facilitados por la Granja de Cuyes de Cieneguilla del Programa de Investigación y Proyección Social en Carnes (PIPS-Carne) de la UNALM. Los animales fueron colocados en jaulas metabólicas individuales, distribuyéndose al azar en dos grupos de cinco animales cada uno.

\section{Forraje Seco de Mucuna}

Se utilizaron semillas de frijol mucuna (Mucuna pruriens) de la variedad moteado, que fueron sembradas en una parcela de $10 \mathrm{~m}^{2}$ del Jardín Agrostológico de la Facultad de Zootecnia, UNALM. Fue cosechado a los seis meses cuando alcanzó el $50 \%$ de floración. El rendimiento forrajero fue de 24 $\mathrm{t} / \mathrm{ha}$. El forraje fresco fue picado, secado en sombra durante siete días y luego fue llevado a estufa a una temperatura de $60-70{ }^{\circ} \mathrm{C}$ durante seis horas. Posteriormente fue molido en un molino «Wiley» de tijeras con malla de $2 \mathrm{~mm}$, obteniéndose el forraje seco del tamaño requerido.
Cuadro 1. Composición de la dieta basal

\begin{tabular}{lc}
\hline Ingredientes & Porcentaje \\
\hline Afrecho & 62.01 \\
Hominy feed & 15.00 \\
Forraje seco de maíz & 10.00 \\
Torta de soya & 7.50 \\
Heno de alfalfa & 4.00 \\
Carbonato de Calcio & 1.00 \\
Fosfato dicálcico & 0.10 \\
Sal & 0.30 \\
Metionina & 0.05 \\
Proapak & 0.04 \\
\hline Total & 100.0 \\
\hline
\end{tabular}

\section{Tratamientos}

Se utilizaron dos dietas: La Dieta 1 $\left(100 \%\right.$ de dieta basal $\left.\left[\mathrm{D}_{\mathrm{B}}\right]\right)$ (Cuadro 1), la cual consistió en alimento balanceado en forma de harina, utilizado en la Granja de Cuyes de la UNALM, a la que se adicionó vitamina C protegida (Rovimix stay-35) a una concentración de $0.60 \mathrm{~g} / \mathrm{kg}$ de alimento. Por otro lado, la Dieta 2 consistió en una mezcla de forraje seco de mucuna $(20 \%)+80 \%$ de la dieta basal $\left(\mathrm{D}_{\mathrm{B}}\right)$.

\section{Experimento}

El experimento constó un periodo de adaptación y otro de colección de heces. El propósito del primer periodo fue acostumbrar a los cuyes al manejo, instalaciones, tipo de alimento y determinar el consumo diario promedio. Esta etapa tuvo una duración de 14 días. Durante los primeros cuatro días se realizó un cambio gradual del alimento, suprimiendo poco a poco el consumo de forraje (chala) a los 10 animales y en el caso de los cuyes con la Dieta 2 se fue aumentando el nivel del forraje seco de mucuna dentro del concentrado, hasta conseguir el nivel requerido (20\%). En los 10 días siguientes del periodo 
de adaptación se determinó el consumo voluntario del alimento cada 24 horas mediante la diferencia del alimento ofrecido menos el residuo.

El periodo de colección de heces tuvo una duración de cinco días, durante el cual también se registró el consumo de alimento. Se realizó la colección total de heces, utilizando las bandejas en forma de embudo de cada jaula metabólica para evitar la contaminación con orina. Las heces de cada animal fueron pesadas diariamente y refrigeradas en bolsas de polietileno individuales, pero que fueron mezcladas al final (pool de heces/animal). Del pool de heces se tomaron muestras $(5 \mathrm{~g})$ para ser llevadas a la estufa $\left(105^{\circ} \mathrm{C}\right.$ por $6 \mathrm{~h}$ ) para determinar la materia seca (MS). Las heces restantes de cada animal fueron secadas y molidas $(2 \mathrm{~mm})$ para el análisis químico proximal.

Se ofreció $80 \mathrm{~g}$ de alimento por día a cada animal. El alimento fue suministrado una vez al día y a la misma hora (09:00). Asimismo, se suministró diariamente agua limpia y fresca ad libitum. Los animales fueron pesados al inicio y al final del experimento. El registro de peso de los animales, alimento ofrecido, alimento no consumido y heces se realizó utilizando una balanza de capacidad de $2 \mathrm{~kg}$ y $0.1 \mathrm{~g}$ de aproximación. Las muestras de la dieta basal, dieta experimental y heces colectadas en la prueba de digestibilidad fueron sometidas al análisis químico proximal utilizando las técnicas establecidas por la AOAC (2012). Para esto, se utilizó una estufa para determinar el contenido de MS, un digestor y equipo Micro kjeldahl para determinar el nitrógeno total (se usó el factor 6.25 para transformar a proteína total), un equipo Soxhlet para la extracción de la grasa y un digestor para determinar la fibra mediante digestión acida y alcalina. La ceniza se obtuvo por incineración en el equipo mufla y el extracto libre de nitrógeno (ELN) se determinó por diferencia.
Con base a los resultados obtenidos de la prueba de consumo, recolección de heces y de los análisis proximales de las dietas y las heces se determinó el coeficiente de digestibilidad aparente (CDA) de los nutrientes, aplicando el método directo para la dieta basal y la dieta experimental. Los coeficientes de digestibilidad del forraje seco de mucuna se determinaron utilizando el método indirecto, mediante las fórmulas descritas por Crampton y Harris (1974). Este método fue aplicado por Valerio (2015) para determinar la energía metabolizable y digestible del gluten de maíz, hominy feed y subproducto de trigo en cuyes, mientras que Clemente et al. (2003) lo utilizaron para evaluar el valor nutricional de Puya llatensis en la alimentación del cuy (Cavia porcellus) y por Sotelo et al. (2016) en la determinación de digestibilidad y energía digestible de leguminosas tropicales.

- Método directo: CDA $(\%)=([$ Nutriente ingerido - Nutriente en heces]/Nutriente ingerido) $* 100$

- Método indirecto: $\mathrm{D}=(100[\mathrm{~T}-\mathrm{B}] / \mathrm{S})+\mathrm{B}$, donde $\mathrm{D}=$ Coeficiente de digestibilidad del alimento en estudio; $B=$ Coeficiente de digestibilidad de la dieta basal; $\mathrm{T}=$ Coeficiente de digestibilidad de la dieta experimental; $\mathrm{S}=$ Nivel de sustitución de la dieta basal por el alimento en estudio $(20 \%)$.

Los cálculos de digestibilidad se realizaron con base al $100 \%$ de MS. Para el cálculo de la energía digestible (ED) se estimó previamente la energía bruta, multiplicando los porcentajes de la fracción proximal de las dietas y de las heces con sus valores calóricos promedios (proteína: $5.7 \mathrm{kcal} / \mathrm{g}$; extracto etéreo: $9.3 \mathrm{kcal} / \mathrm{g}$; ELN y fibra: $4.1 \mathrm{kcal} / \mathrm{g}$ ), según AEC (1978). Luego, en base a su contenido energético se determinó la ED del alimento mediante la fórmula descrita por Crampton y Harris (1974): ED = EB $\left(\mathrm{EH}^{*} \mathrm{Qh}\right) / \mathrm{Ia}$, donde $\mathrm{ED}=$ Energía digestible del alimento (kcal/g); EB = Energía bruta del alimento $(\mathrm{kcal} / \mathrm{g}) ; \mathrm{EH}=$ Energía bruta de las 
Cuadro 2. Composición química de las dietas experimentales

\begin{tabular}{lcccc}
\hline & \multicolumn{2}{c}{ Dieta basal } & \multicolumn{2}{c}{ Dieta experimental } \\
\cline { 2 - 5 } Componentes & $\begin{array}{c}\text { Base } \\
\text { parcialmente } \\
\text { seca }\end{array}$ & Base seca & $\begin{array}{c}\text { Base } \\
\text { parcialmente } \\
\text { seca }\end{array}$ & Base seca \\
\hline Humedad & 11.35 & 0.00 & 11.78 & 0 \\
Materia seca & 88.65 & 100.00 & 88.72 & 100.00 \\
Proté́na cruda & 19.62 & 22.13 & 17.96 & 20.36 \\
Fibra cruda & 7.52 & 8.48 & 11.28 & 12.79 \\
Extracto etéreo & 4.39 & 4.95 & 3.74 & 4.24 \\
Extracto libre de & 51.47 & 58.06 & 49.09 & 55.65 \\
$\quad$ nitrógeno $($ ELN) & & & & \\
Ceniza & 5.65 & 6.37 & 6.14 & 6.96 \\
Materia orgánica & 83.00 & 93.62 & 82.07 & 93.04 \\
Energía bruta $(\mathrm{kcal} / \mathrm{g})$ & & 4.45 & & 4.36 \\
\hline
\end{tabular}

heces $(\mathrm{kcal} / \mathrm{g}) ; \mathrm{Qh}=$ Cantidad de heces producidas por día (g); Ia = Cantidad de alimento ingerido por día (g).

\section{Análisis Estadístico}

Los datos obtenidos por variable fueron resumidos mediante un análisis descriptivo utilizando al promedio como medida de tendencia y la desviación estándar y el coeficiente de variabilidad como medidas de variabilidad.

\section{Resultados y Discusión}

\section{Composición Química}

El análisis químico proximal de la dieta basal y experimental se realizó según las técnicas establecidas por la AOAC (2012), cuyos resultados se muestran en el Cuadro 2.

Los valores obtenidos, tanto para la proteína bruta como para la fibra bruta, en baseseca del forraje seco de mucuna se encuentran dentro de los porcentajes encontrados por Díaz et al. (2002) y Sotelo et al. (2016) en diferentes forrajes tropicales. No obstante, se debe indicar que la composición nutricional se ve afectada por la especie de planta, madurez, cultivo, medio ambiente, insumos (suelo, luz, nutrientes y agua, entre otros) y el manejo (Skerman et al., 1991; Casler et al., 2006). La composición química y la energía bruta en base seca de la leguminosa se presentan en el Cuadro 3.

\section{Digestibilidad Aparente}

Los coeficientes de digestibilidad aparente de los nutrientes del forraje seco de mucuna se presentan en el Cuadro 4. Se precia que el coeficiente de digestibilidad de la MS (66.29\%) es mayor respecto al matarratón (Gliricidia sepium) (49.9\%) determinados en cuyes por Hurtado et al. (2012), a la alfalfa (63.82\%) determinada en conejos por Dihigo et al. (2005), y a la mucuna (65\%) cuando se incluye en $30 \%$ en raciones para corderos (Loyra et al., 2013), pero inferior a la mucuna (73.4\%) y dolico (76.4\%) determinados mediante digestibilidad in vitro con uso de inóculo cecal de conejos alimentados por 30 días con una dieta que incluía el forraje (Caro y Dihigo, 2012) y al 
Cuadro 3. Análisis químico proximal y energía bruta del forraje seco de mucuna (Mucuna pruriens)

\begin{tabular}{lcc}
\hline Componentes & $\begin{array}{c}\text { Base parcialmente seca } \\
(\%)\end{array}$ & $\begin{array}{c}\text { Base seca } \\
(\%)\end{array}$ \\
\hline Humedad & 12.96 & 0 \\
Materia seca & 87.04 & 100.00 \\
Proteína cruda & 12.43 & 14.28 \\
Fibra cruda & 29.14 & 33.48 \\
Extracto etéreo & 1.38 & 1.59 \\
Cenizas & 8.76 & 10.06 \\
Extracto libre de nitrógeno (ELN) & 35.33 & 40.59 \\
Materia orgánica & 78.28 & 89.89 \\
Energía bruta, $1 / \mathrm{g}$ & - & 4.00 \\
\hline
\end{tabular}

trebolillo espadillano (Trifolium dubium) (76.3\%), según Oliva et al. (2015).

De igual manera, el coeficiente de digestibilidad de la materia orgánica - $\mathrm{MO}-$ $(66.13 \%)$ es mayor a las leguminosas tropicales kudzu (Pueraria phaseoloides) (44.4\%), Centrosoma (Centrosema pubescens) (41.6\%), stylo (Stylosanthes guianensis) $(57.4 \%)$ y desmodio (Desmodium ovalifolium) (32.2\%), determinados in vitro (Rosales y Tang, 1996), pero menor a los del trébol encarnado ( $T$. incarnatum $\mathrm{L}$ ), migueliano (Trifolium michelianum), persa ( $T$. resupinatum $\mathrm{L}$. ssp. resupinatum) y vesiculoso (T. vesiculosum Savi), cuyo promedio fue de 72.6\%, según Pereira et al. (2012).

La MO de un forraje hace referencia a la cantidad de MS que este contiene, excluidos los minerales o materiales inorgánicos, los que generalmente son mínimos en los alimentos, pero por ser elementos metálicos tienen elevado peso molecular, lo cual en cierta forma puede repercutir en la digestibilidad de los alimentos (Narváez y Delgado, 2012). Se reconoce que los forrajes vegetativos tienen una mayor calidad nutricional que los pastos maduros, pero también tienen menor rendimiento de MS y alto contenido de humedad,
Cuadro 4. Coeficientes de digestibilidad aparente del forraje seco de mucuna (Mucuna pruriens) en base seca

\begin{tabular}{lc}
\hline Componentes & $\begin{array}{c}\text { Digestibilidad } \\
(\%)\end{array}$ \\
\hline Materia seca & 66.29 \\
Materia orgánica & 66.13 \\
Proteína cruda & 74.02 \\
Extracto etéreo & 60.18 \\
Fibra cruda & 50.82 \\
Ceniza & 73.33 \\
Extracto libre de & 78.22 \\
nitrógeno & \\
\hline
\end{tabular}

por lo que la digestibilidad de MS y MO está relacionada a la edad del forraje al momento del corte (Ribeiro et al., 2014).

El coeficiente de digestibilidad de la proteína cruda (PC) fue $74.02 \%$, superior a la mucuna $(70.8 \%)$ y alfalfa $(70.6 \%)$ determinada en conejos por Dihigo et al. (2005), a las leguminosas tropicales determinados por Sotelo et al. (2016), a la mucuna (62\%) determinada en ovinos con inclusión de un 30\% en la dieta (Loyra et al., 2013), al 
Stylosanthes macrocephala $(56.8 \%)$ y Stylosanthes guianensis (39.9\%), determinadas en ovinos (Villaquiran y Lascano, 1986), debiéndose en parte que las leguminosas están en estado maduro. Por otro lado, este valor fue inferior al de la leguminosa matarratón (91.6\%) (Hurtado et al., 2012), pudiendo deberse a su menor consumo y mayor tiempo de permanencia en el tracto gastrointestinal, lo que aumentaría la digestión por mayor contacto de la digesta con las vellosidades intestinales.

Por lo general el contenido de proteína en los forrajes es elevado cuando son jóvenes y desciende con la madurez, ya que un alto porcentaje de la proteína está ligada a su fibra, lo que afecta su digestión y absorción (Angulo y Rosero, 2018). La digestibilidad se ve limitada por la conformación de la proteína, ya que las proteasas atacan a las proteínas insolubles más lentamente que a las proteínas globulares solubles (Gonzales et al, 2007). También la unión a ciertos metales, lípidos, ácidos nucleicos, celulosa u otros polisacáridos puede limitar parcialmente la digestibilidad, así como como los factores antinutricionales que afectan la digestión y la absorción de aminoácidos (Rubio y Molina, 2016).

El coeficiente de digestibilidad de la fibra cruda - FC - $(50.82 \%)$ fue superior al del kudzu (26.5\%) (Chauca et al., 1978), a las leguminosas tropicales determinados por Sotelo et al. (2016) y la forrajera arbustiva morera (45.2\%) determinadas en cuyes (Meza-Bone et al., 2012). La mayor digestibilidad de la fibra de la mucuna se debe, en parte, a que tiene menor contenido de pared celular y lignina, mayor solubilidad, capacidad buffer y mayor capacidad de retención de agua por parte de la fibra, lo cual favorece la hidrólisis de las enzimas celulasas en el tracto digestivo (Martínez et al., 2008). Sin embargo, tuvo una menor digestibilidad que la leguminosa matarratón $(75.0 \%)$ determinado en cuyes (Hurtado et al., 2012). La digestibilidad de la fibra depende del grado de maduración; es decir, del contenido de la pared celular y el grado de lignificación del forraje. En general, a medida que aumenta la madurez de la planta, disminuye su contenido de proteína y de azúcares solubles y se eleva el contenido de fibra (principalmente celulosa y lignina), lo que causa una disminución gradual en la digestibilidad (Ramírez Orduña et al., 2002; Savon, 2015). Así mismo, el contenido de energía y proteína están relacionados directamente con el desdoblamiento microbiano de la fibra (Narváez y Delgado, 2012).

El $60.18 \%$ de digestibilidad del extracto etéreo del estudio fue superior a la leguminosa matarratón $(15.1 \%)$, determinado por Hurtado et al. (2012), al kudzu (23.9\%) estudiado por Chauca et al. (1978) y a las leguminosas tropicales determinados por Sotelo et al. (2016), la baja digestibilidad puede deberse en parte que en las demás muestras en estudio tienen ceras, suberina y polímeros cerosos, los cuales no se absorben en el tracto gastrointestinal y dificultan la absorción de las grasas saturadas e insaturadas (Van Soest ,1993 y Figueiredo et al., 2019).

El 78.22\% de digestibilidad del ELN fue similar a la digestibilidad reportada para la morera (77.5\%) (Hurtado et al., 2012) y caraca (Erythrina poeppigiana) (76.6\%) (Meza et al., 2012), mientras que fue superior al $73.8 \%$ reportado para kudzu, según Chauca et al. (1978), y a las leguminosas tropicales determinados por Sotelo et al. (2016). El ELN está constituido principalmente por carbohidratos solubles, así como por vitaminas y demás compuestos orgánicos solubles no nitrogenados, los cuales están inversamente relacionados con la cantidad de fibra presente en los forrajes (Savon et al., 2005). La alta digestibilidad del ELN se debe a que la proporción de carbohidratos es mayor que en la fibra, la cual hace que las bacterias del ciego puedan degradar este componente. La composición y alta digestibilidad del ELN reflejan también el valor del coeficiente de digestibilidad aparente encontrada para la materia seca y la energía digestible. 
Cuadro 5. Energía digestible del forraje seco de mucuna (Mucuna pruriens) en base seca

\begin{tabular}{lccc}
\hline Componentes & Basal & Mezcla & $\begin{array}{c}\text { Forraje de } \\
\text { mucuna }\end{array}$ \\
\hline Energía bruta del alimento, kcal/g & 4.45 & 4.36 & - \\
Energía bruta del alimento, kcal/g & 4.16 & 4.15 & - \\
Ingestión de alimento, g/día & 64.16 & 66.06 & - \\
Cantidad de heces, g/día & 17.26 & 18.65 & - \\
Energía digestible, kcal/g & 3.33 & 3.19 & 2.61 \\
\hline
\end{tabular}

\section{Energía Digestible}

La ED para el forraje seco de mucuna fue de $2.61 \mathrm{kcal} / \mathrm{g}$ en base seca (Cuadro 5). Este valor es similar a la ED de la morera (2.66 kcal/g) determinado por Hurtado et al. (2012). Así mismo, muy superior a la ED de las leguminosas tropicales Arachis pintoi $(2.20 \mathrm{kcal} / \mathrm{g})$, Pueraria phaseoloides $(1.6$ $\mathrm{kcal} / \mathrm{g})$, Stylosanthes guianensis $(1.6 \mathrm{kcal} / \mathrm{g})$, Centrosema macrocarpum $(1.4 \mathrm{kcal} / \mathrm{g}) \mathrm{y}$ Erythrina poeppigiana $(1.0 \mathrm{kcal} / \mathrm{g})$ determinados por Sotelo et al. (2016) y a la leguminosa matarratón $(1.9 \mathrm{kcal} / \mathrm{g})$ determinado por Hurtado et al. (2012).

Por otro lado, el contenido de nutrientes digestibles totales (NDT) del forraje de mucuna fue de $61.49 \%$, valor que lo clasifica como un ingrediente fibroso. Este valor es similar al $60.2 \%$ del tallo del maíz chala (Castro y Chirinos, 1997), pero superior al 54.5\% de la broza de quinua (Jara, 2017), e inferior al $7.12 \%$ del rastrojo de maca (Castro et al., 2018).

\section{Conclusiones}

- El forraje seco de mucuna (Mucuna pruriens) tiene coeficientes de digestibilidad entre 51 y $78 \%$ para la materia seca, materia orgánica, proteína cruda, extracto etéreo, extracto libre de nitrógeno, fibra cruda y ceniza y el contenido de nutrientes digestibles totales (NDT) supera el $60 \%$.

- La energía digestible estimada del forraje seco de mucuna fue de $2.61 \mathrm{kcal} / \mathrm{g}$.

- El forraje seco de mucuna puede ser utilizado como ingrediente en la alimentación de cuyes.

\section{Literatura Citada}

1. Alimentation Equilibree de Commentry (AEC). 1978. Animal Feeding. Energy, Aminoacids, Vitamins, Minerals. Document No 4. Francia.

2. Angulo R, Rosero R. 2018. Producción de forraje y calidad nutricional del pasto angleton climacuna (Dichanthium annulatum-Forssk-Stapf) para la producción de heno en La Dorada (Caldas). Rev Prod Anim 30: 10-17.

3. [AOAC] Asociation of Official Analytical Chemists. 2012. Official methods of analysis. $15^{\text {th }}$ ed. Washington DC.

4. Caprita R, Caprita A, Cretescu I, Ursulescu G, Nicu V. 2013. Estimation of in vitro dry matter solubility and protein digestibility of barley grains. Lucrãri atiinpifice-Seria Zootehnie 60: 232-235. 
5. Caro FR. 2010. Efecto de la dosis de gallinaza y la densidad de siembra sobre las características agronómicas de Mucuna Deeringianum Bort. «Mucuna», utilizado como pastura en La Zungaro COCHA - Iquitos. Tesis de Ingeniero Agrónomo. Iquitos, Perú: Univ. Nacional de la Amazonia Peruana. 69 p.

6. Caro Y, Dihigo LE. 2012. Studies of in vitro digestibility of dolicho (Lablab purpureus) and mucuna (Stizolobium niveum) integral meals. Rev Comp Prod Porcina 19: 42-46.

7. Casler MD, Jung HG. 2006. Relationships of fibre, lignin, and phenolics to in vitro fibre digestibility in three perennial grasses. Anim Feed Sci Tech 125: 151-161. doi: 10.1016/ j.anifeedsci.2005.05.015

8. Castro J, Chirinos D. 1997. Nutrición y alimentación de cuyes. Huancayo, Perú: Univ. Nacional del Centro del Perú. 263 p.

9. Castro J, Chirinos D, Calderón J. 2018. Calidad nutricional del rastrojo de la maca (Lepidium peruvianum Chacon) en cuyes. Rev Inv Vet Perú 29: 410-418. doi: 10.15381/rivep.v29i2.13405

10. Chauca L, Saravia J, Agustín A. 1978. Digestibilidad de kudzu, maicillo y gramalote en cuyes. Avances en Investigación - Ministerio de Agricultura y Alimentación 8(1-2): 27-30.

11. Crampton E, Harris L. 1974. Nutrición animal aplicada. Zaragoza, España: Acribia. $756 \mathrm{p}$.

12. Díaz M, González A, Padilla C, Curbelo F. 2002. Caracterización bromatológica de granos y forrajes de las legumonosas temporales Canavalia ensiformis, Lablab purpureus y Stizo-lobium niveum sembradas a finales de la estación lluviosa. Rev Cub Cienc Agric 36: 409-416.

13. Dihigo L, Savon L, Forte C, Martínez M. 2005. Efecto de la sustitución de la alfalfa por niveles de harina de follaje de mucuna (Stizolobium niveum) en dietas para conejos en crecimiento y ceba. Rev Comp Prod Porcina 12: 200-209.
14. Groll H, Lazo H. 2001. Intercambio de experiencias y semillas de agricultores. Asociación de agricultura ecológica. Puerto Maldonado-Perú. Disponible en: https://www.uni-hohenheim.de/ $\sim$ kirchman/mucuna.html

15. Hurtado DI, Nocua S, NarváezSolarte W, Vargas-Sánchez, JE. 2012. Valor nutricional de la morera (Morus sp), matarratón (Gliricidia sepium), pasto india (Panicum maximum) y arboloco (Montanoa quadrangularis) en la alimentación de cuyes (Cavia porcellus). Vet Zootec 6: 56-65.

16. Jara A. 2017. Valor nutricional de la broza de quinua K'iri en cuyes. Tesis de Médico Veterinario Zootecnista. Puno, Perú: Univ Nacional del Altiplano. 71 p.

17. Loyra-Tzab E, Sarmiento-Franco LA, Sandoval-Castro CA, Santos-Ricalde RH. 2013. Nutrient digestibility and metabolizable energy content of Mucuna pruriens whole pods fed to growing pelibuey. Asian Austral J Anim 26: 981 986. doi: 10.5713/ajas.2013.13062

18. Martínez M, Sarmiento L, Santos R, Rodríguez Z, Capetillo C, Savon L, Segura J. 2008. Protein and amino acid apparent ileal digestibility in broilers fed mucuna (Mucuna deeringiana [Bort Merr]) foliage meal in the diet. J Anim Vet Adv 7: 669-672.

19. McDonald P. 2013. Nutrición animal. $7^{\circ}$ ed. España: Acribia. $672 \mathrm{p}$.

20. Meza-Bone GA, Sánchez-Laiño AR, Meza-Chica MA, Meza-Bone CJ, Franco-Suescum NG, AvellanedaCevallos JH, Estupiñán-Véliz KA, et al. 2012. Digestibilidad in vivo de forrajeras arbustivas tropicales para la alimentación de cuyes (Cavia porcellus Linnaeus), en el litoral ecuatoriano. Vet Zootec 6: 8-16.

21. Narváez J, Delgado J. 2012. Valoración de la técnica in vivo aparente para la determinación de la digestibilidad de forrajes en cuyes (Cavia porcellus). Rev Inv Pec 1: 16-24. 
22. Oliva M, Rojas D, Morales A, Oliva C, Oliva M. 2015. Contenido nutricional, digestibilidad y rendimiento de biomasa de pastos nativos que predominan en las cuencas ganaderas de Molinopampa, Pomacochas y Leymebamba, Amazonas, Perú. Scientia Agropec 6: 211-215. doi: 10.17268/sci.agropecu.2015.03.07

23. Pereira-Castro S, Valladares J, Flores $G$, Díaz N, Fernández-Lorenzo B, Resch C, Gonzalez-Arraez A, et al. 2012. Rendimiento y valor nutritivo de nuevas leguminosas anuales como cultivo de invierno en rotaciones forrajeras intensivas en Galicia. Pastos 42: 29-50.

24. Ramírez R, Ramírez RG, López F. 2002. Factores estructurales de la pared celular del forraje que afectan su digestibilidad. UNAL 5: 180-189.

25. Ribeiro G, Teixeira A, Velasco F, Faria $W$, Pereira L, Chaves A, Gonçalves $L$, et al. 2014. Production, nutritional quality and in vitro methane production from Andropogon gayanus grass harvested at different maturities and preserved as hay or silage. Asian Austral J Anim 27: 330-341. doi: 10.5713/ ajas.2013.13161

26. Rosales J, Tang T. 1996. Composición química y digestibilidad de insumos alimenticios de la zona de Ucayali. Folia
Amazónica 8: 13-27. doi: 10.24841/ fa.v8i2.318

27. Rubio LA, Molina E. 2016. Las leguminosas en la alimentación animal. Arbor 192-779): a315. doi: 10.3989/arbor.2016.779n3005

28. Ruiz T, Febles G, Alonso J. 2015. Estudios con leguminosas, un aporte a la ciencia durante los cincuenta años del Instituto de Ciencia Animal. Cuban J Agr Sci 49: 233-241.

29. Savon L. 2015. Physiological aspects of the use of non-traditional feeds for nonruminant species. Cuban J Agr Sci 49: $250-278$

30. Savon L, Gutierrez O, Ojeda F, Scull I. 2005. Harinas de follajes tropicales: una alternativa potencial para la alimentación de especies monogástricas. Pastos y Forrajes 28: 69-79.

31. Skerman P, Cameron DG, Riveros F. 1991. Leguminosas forrajeras tropicales. Roma: FAO.

32. Sotelo A, Contreras $C$, Norabuena $E$ Castañeda, R, Van Heurck M, Bullón L. 2016. Digestibilidad y energía digestible de cinco leguminosas forrajeras tropicales. Rev Soc Quím Perú 82: 306-314.

33. Villaquiran M, Lascano C. 1986. Caracterización nutritiva de cuatro leguminosas forrajeras tropicales. Pasturas Tropicales 8: 2-7. 